\title{
Targeting Hyperglycaemia with Anti-Obesity Drugs: Time for a Paradigm Shift?
}

\author{
Andrew J. Krentz $\cdot$ Marcus Hompesch
}

Published online: 5 October 2013

(C) Springer International Publishing Switzerland 2013

Excess adiposity, arising from a chronic imbalance of energy intake relative to expenditure, is regarded as a hallmark of type 2 diabetes. It is well established that whole-body insulin resistance results from the acquisition of surplus adipose tissue mass. In turn, insulin resistance is implicated in the deleterious cardiometabolic profile that is characteristic of type 2 diabetes [1]. Voluntary decreases in body fat mass lead to improvements in carbohydrate and lipid metabolism together with reductions in blood pressure and markers of inflammation [1,2]. Accordingly, current international treatment guidelines for type 2 diabetes advocate reducing hyperglycemia using non-pharmacological means wherever possible [3, 4].

Beyond the setting of closely supervised clinical trials only a small minority of subjects are able to achieve and sustain degrees of weight loss sufficient to avoid the need for any glucose-lowering medication [2]. Accordingly, a glucose-lowering drug, or a combination of drugs, is chosen from among classes with disparate mechanisms but broadly similar efficacy. Some widely used glucose-lowering drugs-sulphonylureas, thiazolidinediones, and insulin—work against the best efforts of the recipient by promoting weight gain [5]. Newer agents of diabetes drugs that have neutral (e.g. dipeptidyl peptidase-4 inhibitors) or favorable (glucagon-like peptide-1 receptor agonists, sodium-glucose cotransporter-2 inhibitors) effects on body weight avoid this difficulty. Injectable glucagon-like peptide-1 receptor agonists have broad potential as weightreducing agents that also improve cardiovascular risk

A. J. Krentz $(\bowtie) \cdot$ M. Hompesch

Profil Institute for Clinical Research, 855 3rd Ave, Suite 4400,

Chula Vista, CA 91911, USA

e-mail: andrew.krentz@profilinstitute.com

URL: http://www.profilinstitute.com factor profiles [6]. However, at present, agents in this class are approved only for the treatment of type 2 diabetes.

Given the pivotal role of obesity in the pathogenesis of diabetes it is perhaps surprising that drugs licensed primarily for weight reduction are not more widely considered as first- or second-line pharmacological agents for type 2 diabetes accompanied by obesity. No compelling rationale exists that would exclude drugs designed as weightreducing agents as alternative or adjunctive pharmacotherapy in this scenario. However, clinical guidelines for managing type 2 diabetes do not include weight-loss drugs in treatment algorithms. Anti-obesity drugs that have been shown to improve glucose control in subjects with type 2 diabetes include orlistat [7], sibutramine [8], and rimonabant [9]. The latter two agents are no longer available, having been withdrawn because of risk-benefit profiles that were deemed to be unacceptable. While neither drug was licensed for the treatment of hyperglycaemia per se, the presence of diabetes as a co-morbidity permitted their use in patients with a body mass index (BMI) greater than $27 \mathrm{~kg} / \mathrm{m}^{2}$.

Two new centrally acting drugs developed to assist weight reduction have recently become available. Current evidence suggests that both may be of value in managing hyperglycaemia and obesity-associated cardiovascular risk factors such as hypertension. A proprietary combination of phentermine/topiramate $\left(\right.$ Qysmia $^{\circledR}$; Vivus Inc., Mountain View, CA, USA) was approved in 2012 by the US Food and Drug Administration (FDA) as an addition to a reduced-calorie diet and exercise for chronic weight management. This once-daily capsule contains phentermine, a sympathomimetic amine anorectic, and topiramate extended-release. The latter agent is indicated for certain forms of seizures but can produce significant weight loss as monotherapy. The combination capsule contains relatively 
low doses of each agent to address concerns about tolerability. FDA approval came in the form of a Risk Evaluation and Mitigation Strategy (REMS) that initially restricted supply to specialty mail-order pharmacies. This cautious approach aimed to ensure that prescribers and women of reproductive potential were informed of the risk of orofacial cleft defects in fetuses exposed to treatment during the first trimester of pregnancy. The efficacy of phentermine/ topiramate has been demonstrated in clinical trials. In the CONQUER trial, overweight or obese patients with two or more comorbidities (diabetes or pre-diabetes, hypertension, dyslipidaemia, or abdominal obesity) were randomized to (i) a lower-dose combination of the study drug $(7.5 \mathrm{mg}$ phentermine/46 mg topiramate, 498 subjects); (ii) a higher dose (15 mg phentermine/92 mg topiramate, 995 subjects); or (iii) placebo [10]. At 56 weeks, subjects randomized to the higher-dose combination had the greatest mean weight loss, approximately $2 \mathrm{~kg}$ more than subjects taking the lower-dose combination; the placebo group lost a mean of $1.4 \mathrm{~kg}$. In a recently reported post hoc analysis of data from CONQUER, phentermine/topiramate was associated with a reduction in hemoglobin $(\mathrm{Hb}) \mathrm{A}_{1 \mathrm{c}}$ of 0.4 versus 0.1 percentage points with placebo $(p<0.05)$ among 388 participants with previously diagnosed diabetes or elevated fasting glucose levels [11]. Moreover, fewer patients with diabetes receiving active treatment increased the number of glucose-lowering medications (4 vs. $15 \%$ ) taken during the 56-week study. As observed in non-diabetic participants, subjects with type 2 diabetes randomized to phentermine/topiramate lost significantly more weight than placebo-treated controls, with a larger proportion losing more than $10 \%$ of their body weight (40 and 27 vs. $6 \%$, for high dose, low dose, and placebo, respectively; $p<0.0001$ ). Phentermine/topiramate is currently in phase II of clinical development for the treatment of type 2 diabetes.

Another new anti-obesity drug with glucose-lowering effects-lorcaserin (Belviq ${ }^{\circledR}$; Arena Pharmaceuticals, San Diego, CA, USA) - became available in the US in 2012 [12]. Lorcaserin, a novel selective agonist of the 5-hydroxytryptamine (2C) receptor, is purported to reduce weight by decreasing food consumption and increasing satiety [13]. Of note, preclinical and clinical studies indicate lorcaserin, unlike fenfluramine and dexfenfluramine, which also act on serotonin metabolism, is not associated with cardiac valvulopathy or risk of pulmonary hypertension $[14,15]$. Lorcaserin is approved for chronic weight loss management in adults as an adjunct to diet and exercise. The drug may be used in subjects with a BMI of more than $30 \mathrm{~kg} / \mathrm{m}^{2}$, or a BMI of more than $27 \mathrm{~kg} / \mathrm{m}^{2}$ in the presence of one or more weight-related comorbidities such as type 2 diabetes, hypertension, or dyslipidaemia [16]. Safety issues associated with lorcaserin include the possibility of serotonin syndrome or neuroleptic malignant syndrome when co-administered with other serotonergic or anti-dopaminergic agents, cognitive impairment, and psychiatric disorders. The Behavioral Modification and Lorcaserin for Obesity and Overweight Management in Diabetes Mellitus (BLOOM-DM) study evaluated the efficacy and safety of lorcaserin for weight loss in patients with type 2 diabetes [17]. This 52-week, placebo-controlled trial enrolled 604 patients who were randomized 1:1:1 to placebo, lorcaserin $10 \mathrm{mg}$ once daily, or lorcaserin $10 \mathrm{mg}$ twice daily, respectively. The mean $( \pm \mathrm{SD})$ age of the participants was $52.7 \pm 8.7$ years. Mean BMI was $36.0 \pm 4.5 \mathrm{~kg} / \mathrm{m}^{2}$. The majority of subjects were taking glucose-lowering medication, principally metformin (92\%) and/or a sulphonylurea (50\%). More patients lost $\geq 5 \%$ of their body weight with lorcaserin twice daily $(37.5 \% ; p<0.001)$ or lorcaserin once daily $(44.7 \%$; $p<0.001)$ compared with placebo $(16.1 \%)$. $\mathrm{HbA}_{1 \mathrm{c}}$ decreased $0.9 \pm 0.06$ with lorcaserin twice daily and $1.0 \pm 0.09$ with lorcaserin once daily, compared with $0.4 \pm 0.06$ with placebo $(p<0.001$ for each).

What are the prospects for either phentermine/topiramate or lorcaserin becoming accepted as adjunctive pharmacotherapy for type 2 diabetes? The aforementioned clinical trials provide compelling evidence that both have clinically relevant effects on glycaemia. The reductions in glycated haemoglobin observed in those trials, if sustained, would be expected to reduce the risk of vascular complications. However, such benefits must be weighed against uncertainties about long-term safety [18]. Arriving so shortly after the demise of rimonabant and sibutramine, the safety profiles of phentermine/topiramate and lorcaserin are under close scrutiny. In approving the combination of phentermine/topiramate, an FDA panel strongly recommended that a large morbidity and mortality study be initiated. Based on the results of short-term studies, lorcaserin seems to be well tolerated with no cardiovascular safety signals $[19,20]$. However, in granting marketing approval, the FDA stipulated that lorcaserin was required to undergo a long-term cardiovascular safety study to assess the risk of myocardial infarction and stroke. Regulatory authorities became highly sensitized to the risk-to-benefit ratios of new glucose-lowering drugs in the wake of the rosiglitazone debacle [21, 22]. However, the safety record of antiobesity agents could hardly be said to be less dismal than the recent experience with diabetes drugs.

Incorporating weight-loss drugs into treatment algorithms for type 2 diabetes presents some challenges, not least in shifting the mindset of prescribers conditioned by decades of convention. This said, a precedent was set in 2009 when a bile acid sequestrant-colesevelam-was positioned within the American Association of Clinical Endocrinologists/American College of Endocrinology 
(AACE/ACE) type 2 diabetes consensus panel glycemic control guidelines [23]. This move was followed with a recommendation from the AACE that obesity management, including medication alongside lifestyle and surgical options, be considered as first-line treatment for pre-diabetes, i.e. impaired fasting glucose or impaired glucose tolerance [5]. In the pursuit of a more personalized approach to managing type 2 diabetes [24], bringing novel weight-reducing agents into treatment algorithms will inevitably add an additional layer to what has already become a complex therapeutic landscape.

With mounting evidence of efficacy allied to the need for effective and safe new therapeutic approaches, the stage now seems set for wider application of novel anti-obesity drugs in the management of type 2 diabetes. However, additional data are required to support this strategy. Future trials should compare the glucose-lowering efficacy and safety profiles of these novel weight-reducing drugs directly against each other and also against established and newer classes of drugs approved for type 2 diabetes. Additional developments in anti-obesity pharmacotherapy are anticipated [16] that may usefully expand the options for achieving glycemic targets in type 2 diabetes.

Acknowledgments We thank Prof. Steven Smith of the SanfordBurnham Medical Research Institute, Orlando, FL, USA, for helpful discussions.

Competing Interests Profil Institute for Clinical Research performs early phase studies of new therapies for diabetes and obesity.

Funding No sources of funding were used to prepare this editorial.

\section{References}

1. Klein S, Sheard NF, Pi-Sunyer X, et al. Weight management through lifestyle modification for the prevention and management of type 2 diabetes: rationale and strategies: a statement of the American Diabetes Association, the North American Association for the Study of Obesity, and the American Society for Clinical Nutrition. Diabetes Care. 2004;27:2067-73.

2. Taylor R. Type 2 diabetes: etiology and reversibility. Diabetes Care. 2013;36:1047-55.

3. Guidlines for the management of type 2 diabetes. 2012. http:// www.idf.org. Accessed 11 July 2013.

4. Inzucchi SE, Bergenstal RM, Buse JB, et al. Management of hyperglycemia in type 2 diabetes: a patient-centered approach: position statement of the American Diabetes Association (ADA) and the European Association for the Study of Diabetes (EASD). Diabetes Care. 2012;35:1364-79.

5. Garber AJ, Abrahamson MJ, Barzilay JI, et al. AACE comprehensive diabetes management algorithm 2013. Endocr Pract. 2013;19:327-36.

6. Vilsboll T, Christensen M, Junker AE, Knop FK, Gluud LL. Effects of glucagon-like peptide-1 receptor agonists on weight loss: systematic review and meta-analyses of randomised controlled trials. BMJ. 2012;344:d7771.

7. Jacob S, Rabbia M, Meier MK, Hauptman J. Orlistat $120 \mathrm{mg}$ improves glycaemic control in type 2 diabetic patients with or without concurrent weight loss. Diabetes Obes Metab. 2009;11:361-71.

8. Vettor R, Serra R, Fabris R, Pagano C, Federspil G. Effect of sibutramine on weight management and metabolic control in type 2 diabetes: a meta-analysis of clinical studies. Diabetes Care. 2005;28:942-9.

9. Scheen AJ, Finer N, Hollander P, Jensen MD, Van Gaal LF. Efficacy and tolerability of rimonabant in overweight or obese patients with type 2 diabetes: a randomised controlled study. Lancet. 2006;368:1660-72.

10. Gadde KM, Allison DB, Ryan DH, et al. Effects of low-dose, controlled-release, phentermine plus topiramate combination on weight and associated comorbidities in overweight and obese adults (CONQUER): a randomised, placebo-controlled, phase 3 trial. Lancet. 2011;377:1341-52.

11. Astrup AV, Oppert J-M, Peterson CA. Weight loss and improvements in cardiometabolic outcomes with extendedrelease phentermine/topiramate treatment in overweight/obese subjects with type 2 diabetes. Diabetologia 2012;55(suppl 1):S48.

12. Redman LM, Ravussin E. Lorcaserin for the treatment of obesity. Drugs Today. 2010;46:901-10.

13. Nigro SC, Luon D, Baker WL. Lorcaserin: a novel serotonin $2 \mathrm{C}$ agonist for the treatment of obesity. Curr Med Res Opin. 2013;29:839-48.

14. Hoy SM. Lorcaserin: a review of its use in chronic weight management. Drugs. 2013;73(5):463-73.

15. Bai B, Wang Y. The use of lorcaserin in the management of obesity: a critical appraisal. Drug Des Dev Ther. 2010;5:1-7.

16. Gallwitz B. Novel oral anti-obesity agents: new perspectives with lorcaserin? Drugs. 2013;73:393-5.

17. O'Neil PM, Smith SR, Weissman NJ, et al. Randomized placebocontrolled clinical trial of lorcaserin for weight loss in type 2 diabetes mellitus: the BLOOM-DM study. Obesity. 2012;20:1426-36.

18. Kenkre J, Tan T, Bloom S. Treating the obese diabetic. Expert Rev Clin Pharmacol. 2013;6:171-83.

19. Smith SR, Prosser WA, Donahue DJ, Morgan ME, Anderson CM, Shanahan WR. Lorcaserin (APD356), a selective 5-HT(2C) agonist, reduces body weight in obese men and women. Obesity. 2009; 17:494-503.

20. Chan EW, He Y, Chui CS, Wong AY, Lau WC, Wong IC. Efficacy and safety of lorcaserin in obese adults: a meta-analysis of 1-year randomized controlled trials (RCTs) and narrative review on short-term RCTs. Obes Rev. 2013;14(5):383-92.

21. Krentz AJ. Rosiglitazone: trials, tribulations and termination. Drugs. 2011;71:123-30.

22. Hiatt WR, Kaul S, Smith RJ. The cardiovascular safety of diabetes drugs: insights from the rosiglitazone experience. $\mathrm{N}$ Engl $\mathrm{J}$ Med. Epub 2 Sep 2013

23. Rodbard HW, Jellinger PS, Davidson JA, et al. Statement by an American Association of Clinical Endocrinologists/American College of Endocrinology consensus panel on type 2 diabetes mellitus: an algorithm for glycemic control. Endocr Pract. 2009;15:540-59.

24. Raz I, Riddle MC, Rosenstock J, et al. Personalized management of hyperglycemia in type 2 diabetes: reflections from a diabetes care editors' expert forum. Diabetes Care. 2013;36:1779-88. 